\title{
Curcumin enhances the lung cancer chemopreventive efficacy of phospho-sulindac by improving its pharmacokinetics
}

\author{
KA-WING CHENG, CHI C. WONG, GEORGE MATTHEOLABAKIS, GANG XIE, LIQUN HUANG and BASIL RIGAS \\ Division of Cancer Prevention, Department of Medicine, Stony Brook University, Stony Brook, NY 11794-8173, USA
}

Received March 16, 2013; Accepted April 29, 2013

DOI: 10.3892/ijo.2013.1995

\begin{abstract}
Phospho-sulindac (PS) is a safe sulindac derivative with promising anticancer efficacy in colon cancer. We evaluated whether its combination with curcumin could enhance the efficacy in the treatment of lung cancer. Curcumin, the principal bioactive component in turmeric, has demonstrated versatile capabilities to modify the therapeutic efficacy of a wide range of anticancer agents. Here, we evaluated the effect of co-administration of curcumin on the anticancer activity of PS in a mouse xenograft model of human lung cancer. Curcumin enhanced the cellular uptake of PS in human lung and colon cancer cell lines. To assess the potential synergism between curcumin and PS in vivo, curcumin was suspended in $10 \%$ Tween- 80 or formulated in micellar nanoparticles and given to mice by oral gavage prior to the administration of PS. Both formulations of curcumin significantly improved the pharmacokinetic profiles of PS, with the $10 \%$ Tween- 80 suspension being much more effective than the nanoparticle formation. However, curcumin did not exhibit any significant modification of the metabolite profile of PS. Furthermore, in a mouse subcutaneous xenograft model of human lung cancer, PS $(200 \mathrm{mg} / \mathrm{kg})$ in combination with curcumin $(500 \mathrm{mg} / \mathrm{kg})$ suspended in $10 \%$ Tween-80 (51\% inhibition, $\mathrm{p}<0.05)$ was significantly more efficacious than PS plus micelle curcumin (30\%) or PS $(25 \%)$ or curcumin alone (no effect). Consistent with the improved pharmacokinetics, the combination treatment group had higher levels of PS and its metabolites in the xenografts compared to PS alone. Our results show that curcumin substantially improves the pharmacokinetics of PS leading to synergistic inhibition of the growth of human lung cancer xenografts, representing a promising drug combination.
\end{abstract}

\section{Introduction}

Lung cancer is the leading cause of cancer-related deaths worldwide. Despite advances in early detection and chemo-

Correspondence to: Professor Basil Rigas, Division of Cancer Prevention, Stony Brook University, HSC, T-17 Room 080, Stony Brook, NY 11794-8173, USA

E-mail: basil.rigas@stonybrookmedicine.edu

Key words: phospho-sulindac, curcumin, lung cancer, cellular uptake, pharmacokinetics therapy, its prognosis is generally poor, with a 5-year survival of approximately $15 \%$. Given a lack of effective therapeutics, the use of chemopreventive agents that abrogate lung carcinogenesis represents a promising approach for controlling this disease.

Compelling evidence has emerged that non-steroidal antiinflammatory drugs (NSAIDs) can reduce the incidence of various cancers and limit metastatic disease (1-3). However, the chronic use of NSAIDs is associated with significant gastrointestinal and renal toxicities. To reduce toxicity and enhance the efficacy of conventional NSAIDs, our group has developed novel phospho-derivatives of NSAIDs. One such derivative is phospho-sulindac (PS, OXT-328), which is efficacious in the prevention and treatment of colon and breast cancer in preclinical models (4-7) and shows a favorable safety profile (5). In contrast, PS as a single agent was ineffective in the treatment of human lung cancer xenografts (8), which prompted us to develop more potent PS-based therapy incorporating other anticancer agents. Here, we describe the combination of PS with curcumin for the prevention of lung cancer.

Curcumin, the principal bioactive component in turmeric, exhibits antitumorigenic activities (9-11). In pre-clinical models of lung cancer, however, curcumin as a single agent has demonstrated poor efficacy $(<30 \%)(12)$; and according to one report, it may even promote Kras-driven lung tumorigenesis in mice (13). On the other hand, curcumin significantly potentiates the antitumor activity of sulindac, docetaxel and gefitinib in animal models (14-16). Curcumin can also overcome resistance to anticancer drugs such as paclitaxel, thalidomide and bortezomib $(17,18)$. Thus, curcumin is a versatile chemosensitizer for mechanistically diverse anticancer agents.

Here, we demonstrate that curcumin potentiates the anticancer efficacy of PS in human non-small cell lung cancer (NSCLC) cells and that such a combination synergistically inhibits the growth of A549 xenografts in mice. These findings suggest that PS plus curcumin is a promising combination for the prevention of NSCLC.

\section{Materials and methods}

Reagents. Phospho-sulindac (OXT-328) was a gift from Medicon Pharmaceuticals, Inc., Setauket, NY, USA. Cell culture reagents were purchased from Cellgro (Herndon, VA, USA). Other reagents, unless otherwise stated, were obtained from Sigma-Aldrich (St. Louis, MO, USA). 
Cell culture. Human NSCLC (A549), breast (MCF-7 and MDA-MB-231), colon (SW480) and pancreatic (MIAPaCa-2) cancer cell lines were obtained from American Type Culture Collection (ATCC) and maintained in the recommended culture media containing $10 \%$ fetal bovine serum and penicillin/streptomycin. All experiments were performed with cells between passages 1 and 10 .

Curcumin formulation. Polymeric nanoparticles of poly( $\varepsilon$ caprolactone) $(11,000)$-polyethylene glycol $(5,000)$ with entrapped curcumin were prepared according to the nanoprecipitation-solvent displacement method $(19,20)$. Briefly, $50 \mathrm{mg}$ of polymer and $5 \mathrm{mg}$ of curcumin were dissolved in $1 \mathrm{ml}$ of acetone and the solution was added dropwise to $2 \mathrm{ml}$ of water under constant stirring. The organic solvent was allowed to slowly evaporate under reduced pressure and the resulting suspension was centrifuged to remove aggregates and drug precipitates. Curcumin concentration was determined using HPLC. Ten minutes after diluting the suspension in water, we determined the size and zeta potential of the nanoparticles using Dynamic Light Scattering (Zeta-Plus Brookhaven instrument, Holtsville, NY, USA). Particle size was also determined using transmission electron microscopy. Curcumin loading in the nanoparticles was $8 \pm 0.3 \%$. The mean nanoparticle size was $45.2 \mathrm{~nm}$ and their polydispersity index was $0.271 \pm 0.005$.

Cell growth inhibition assays. Cell viability was determined by a modified colorimetric assay using 3-[4,5-dimethylthiazol2-yl]-2,5-diphenyltetrazolium bromide (MTT). Briefly, A549 cells seeded in 96-well plates were treated with different concentrations of PS for $24 \mathrm{~h}$ with or without pretreatment with curcumin for $3 \mathrm{~h}$. The culture medium was removed and replaced with $100 \mu \mathrm{l}$ complete medium containing $0.5 \mathrm{mg} / \mathrm{ml}$ MTT. Following 4-h incubation at $37^{\circ} \mathrm{C}, 100 \mu 1$ of a solution containing $10 \%$ SDS and $0.01 \mathrm{~N} \mathrm{HCl}$ was added. The plate was incubated and gently mixed until MTT formazan crystals were dissolved. Absorbance at $570 \mathrm{~nm}$ was measured on a microplate reader and $\mathrm{IC}_{50}$ was calculated after subtraction of blank values.

Apoptotic cell death analysis. After drug treatment, cells were trypsinized, washed once with PBS and stained with Annexin V/propidium iodide. Percentage of apoptotic cells was determined by flow cytometry on a FACSCalibur.

Cellular uptake assay. For the uptake study, cells were seeded into 12 -well plates at a cell density of $2.5 \times 10^{5}$ per well. After overnight incubation, uptake experiments were carried out using serum-free media. After washing the monolayer twice, the cells were pre-incubated for $15 \mathrm{~min}$ with serum-free media with or without transporter inhibitors. PS was then added and incubated for $1 \mathrm{~h}$. At the end of the incubation, the medium was quickly aspirated. The cells were washed twice, each with $2 \mathrm{ml}$ of ice-cold transport buffer containing $0.2 \%$ bovine serum albumin (BSA). Finally, the cells were rinsed with $0.5 \mathrm{ml}$ of ice-cold transport buffer without BSA. Cells were collected with $0.5 \mathrm{ml}$ of $50 \%$ methanol. Extraction was performed by sonication for $5 \mathrm{~min}$ followed by the addition of $0.5 \mathrm{ml}$ of ice-cold methanol and centrifuged at 17,000 $\mathrm{xg}$ for $12 \mathrm{~min}$. The supernatant was collected and analyzed by HPLC. The protein pellet was re-dissolved in $0.1 \mathrm{~N} \mathrm{NaOH}$ and the protein content was determined by the Bradford assay. All the uptake values were corrected against protein content.

Pharmacokinetic studies in mice. This and subsequent animal studies were approved by the Institutional Animal Care and Use Committee (IACUC) of Stony Brook University. Mice $(\mathrm{n}=2)$ were given a single dose of the following treatments: i) PS $200 \mathrm{mg} / \mathrm{kg}$; ii) PS $200 \mathrm{mg} / \mathrm{kg}$ plus curcumin $500 \mathrm{mg} /$ $\mathrm{kg}$, in $10 \%$ Tween-80; and iii) PS $200 \mathrm{mg} / \mathrm{kg}$ plus micellar curcumin $500 \mathrm{mg} / \mathrm{kg}$. At designated time-points, mice were euthanized by $\mathrm{CO}_{2}$ inhalation and blood was collected and immediately centrifuged. The resulting plasma was deproteinized by immediately mixing it with $2.5 \mathrm{x}$ volumes of acetonitrile. The deproteinized samples were analysed by HPLC as described below.

A549 xenografts. Female nude mice 6-7 weeks old were purchased from Harlan Sprague-Dawley, Indianapolis, IN, USA. At 7-8 weeks of age, four groups of mice ( $n=6$ per group) were pre-treated for 3 days with: i) vehicle; ii) PS $200 \mathrm{mg} /$ $\mathrm{kg} / \mathrm{d}$; iii) curcumin $500 \mathrm{mg} / \mathrm{kg} / \mathrm{d}$; and iv) PS $200 \mathrm{mg} / \mathrm{kg} / \mathrm{d}$ plus curcumin $500 \mathrm{mg} / \mathrm{kg} / \mathrm{d}$. Then, the mice were inoculated subcutaneously into both flanks with A459 cells $\left(2 \times 10^{6}\right.$ each) suspended in $100 \mu \mathrm{l}$ complete F12K medium: Matrigel Matrix gel (BD Biosciences, San Jose, CA, USA) (1:1, v/v). The treatment was resumed one day after tumor implantation and continued daily until the end of the study. The tumors were measured twice a week with a digital microcaliper and tumor volumes were calculated using the formula: tumor volume $=$ [length $\mathrm{x}$ width $\mathrm{x}$ (length + width/2) $\mathrm{x} 0.56$ ]. At the end of the experiment, the animals were sacrificed and their tumors were removed. The levels of PS and its metabolites in the tumors were determined by HPLC (21).

HPLC analysis. The HPLC system consisted of a Waters Alliance 2695 Separations Module equipped with a Waters 2998 photo-diode array detector (Waters, Milford, MA, USA) and a Thermo BDS Hypersil C18 column (150x4.6 mm, particle size $3 \mu \mathrm{m}$ ) (Thermo Fisher Scientific, Waltham, MA, USA). The mobile phase consisted of a gradient between solvent A [(trifluoroacetic acid, acetonitrile, $\mathrm{H}_{2} \mathrm{O}$ (0.1:4.9:95, $\mathrm{v} / \mathrm{v} / \mathrm{v})]$ and $100 \%$ acetonitrile.

Statistical analyses. Data are expressed as mean \pm SEM. Statistical analyses were performed by ANOVA. P-values $<0.05$ were considered statistically significant.

\section{Results}

Curcumin synergizes with PS in inhibiting the growth of lung cancer cells in vitro. Pretreatment with curcumin sensitizes A549 lung cancer cells to the cytotoxic effect of PS. As shown in Fig. 1A, pretreatment of A549 cells with non-cytotoxic levels of curcumin $100 \mu \mathrm{M}$ enhanced the cytotoxicity of PS. Following treatment with $100 \mu \mathrm{M}$ curcumin, reductions of cell viability were as follows: PS $80 \mu \mathrm{M}$ alone, $30 \%$; curcumin alone, $4 \%$; and PS plus curcumin, $60 \%$. A similar synergistic effect was also observed in the induction of apoptosis. After 20-h incubation, the percentage of apoptotic cells treated with 
A

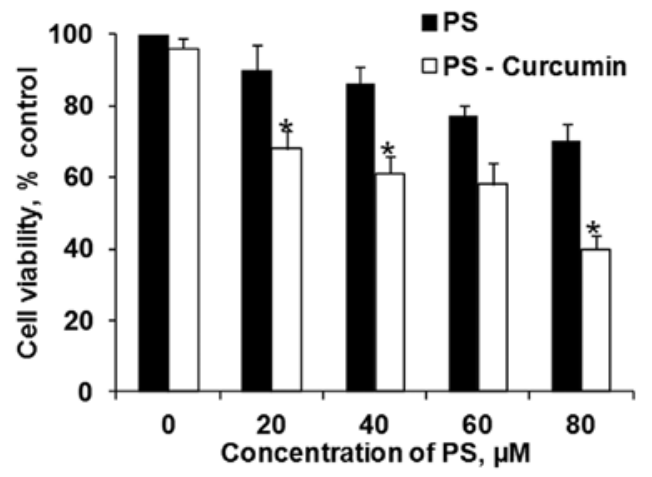

B

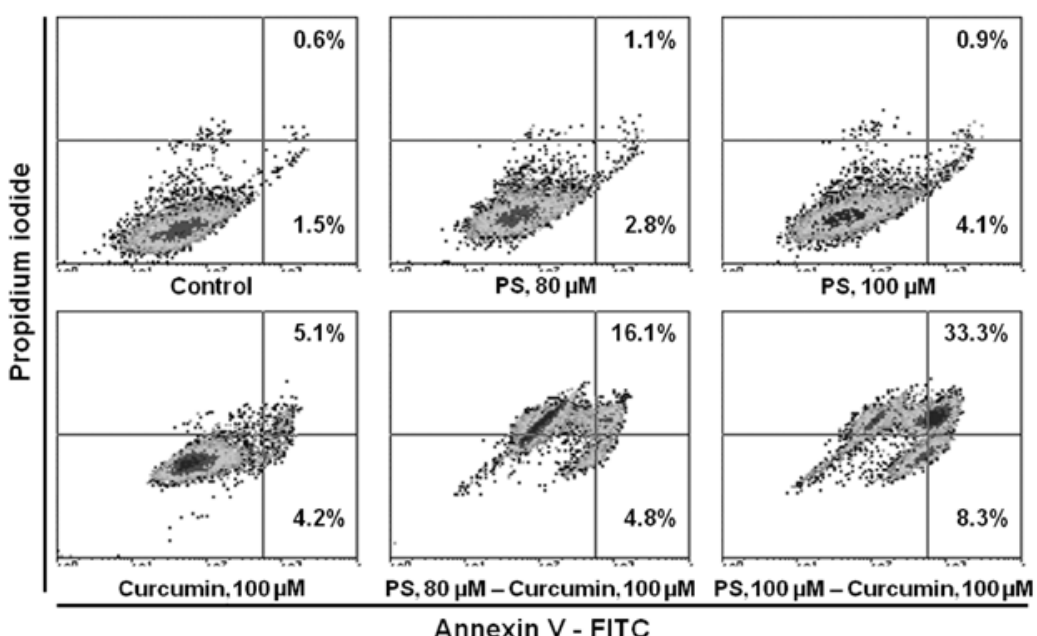

Figure 1. PS and curcumin synergistically inhibit the growth of A549 lung cancer cells in vitro. (A) Percent cell viability of A549 cells following treatment with PS alone $(20-80 \mu \mathrm{M})$ or in combination with curcumin $(100 \mu \mathrm{M})$ pretreatment. "p $<0.05$ compared to control. (B) A549 cells were treated with PS (80 or $100 \mu \mathrm{M})$ alone or in combination with curcumin $(100 \mu \mathrm{M})$. Cells were stained with Annexin V/PI and analyzed by flow cytometry and the percentages of cell populations are indicated in each quadrant. Annexin V (+) cells are apoptotic.

80 and $100 \mu \mathrm{M}$ PS with curcumin $100 \mu \mathrm{M}$ was 20.9 and $41.6 \%$, respectively, compared to 3.9 and $5.0 \%$ with 80 and $100 \mu \mathrm{M}$ PS alone. These findings establish that curcumin potentiates the cytotoxic activity of PS in lung cancer cells.

Curcumin enhances the cellular uptake of PS in cancer cells. Since curcumin is known to synergize with other compounds and to also inhibit cellular efflux transporters, we reasoned that curcumin might have synergistic activity with PS through an effect on ATP-binding cassette (ABC) transporters which are implicated in the cellular efflux of xenobiotics, such as anticancer drugs. Isoform-specific inhibitors of ABC transporters enable better understanding of the role of individual $\mathrm{ABC}$ transporter(s) in the efflux of a drug (22). To identify specific ATP transporters involved in the efflux of PS, changes in the cellular levels of PS were evaluated in the presence of isoform-specific inhibitors. In this study, the involvement of efflux transporters was studied using inhibitors of multidrug resistance proteins (MRPs; MK571), breast cancer resistance protein (BCRP; Ko143) and P-glycoprotein (P-gp; verapamil).

In A549 cells, co-incubation of PS with MK571 resulted in a $51 \%$ increase in the intracellular accumulation of PS (Fig. 2A). Ko143 and verapamil had no effect on the uptake of PS in A549 cells. In SW480 cells, MK571 and verapamil, but not Kol43, enhanced the cellular accumulation of PS by 30 and $67 \%$, respectively (Fig. 2A). It appeared that multidrug resistance proteins (MRPs) are involved in PS efflux in both A549 and SW480 cells; while P-glycoprotein (P-gp) is only involved in the efflux of PS in SW480. BCRP, on the other hand, has little impact on the efflux of PS. Thus, the specific $\mathrm{ABC}$ transporter(s) involved in the efflux of PS is cell-line dependent.

Given that curcumin can inhibit efflux transporters including MRPs and P-gp, we explored the effect of curcumin on the uptake of PS in A549 and SW480 cells. The effect of curcumin ( 5 and $25 \mu \mathrm{M})$ on the accumulation of PS is shown in Fig. 1B. At 5 and $25 \mu \mathrm{M}$, curcumin increased the intracellular levels of PS in A549 cells by 23 and 60\%, respectively. Similarly, curcumin also increased the uptake of PS in SW480 cells by $37 \%$ at $5 \mu \mathrm{M}$ and $54 \%$ at $25 \mu \mathrm{M}$. Therefore, co-incubation with curcumin recapitulated the effect of transporter inhibitors. Since curcumin is an inhibitor of MRPs and P-gp, these findings suggest that curcumin may enhance PS accumulation via inhibition of efflux transports in these cancer cell lines.

PS and curcumin synergistically inhibit the growth of A549 xenografts in mice. We investigated the antitumor efficacy of PS, curcumin or their combination in subcutaneous xenografts of A549 human lung cancer cells in nude mice. PS and curcumin, when given alone, did not significantly inhibit the growth of A549 xenografts (Fig. 3A). PS alone produced a 

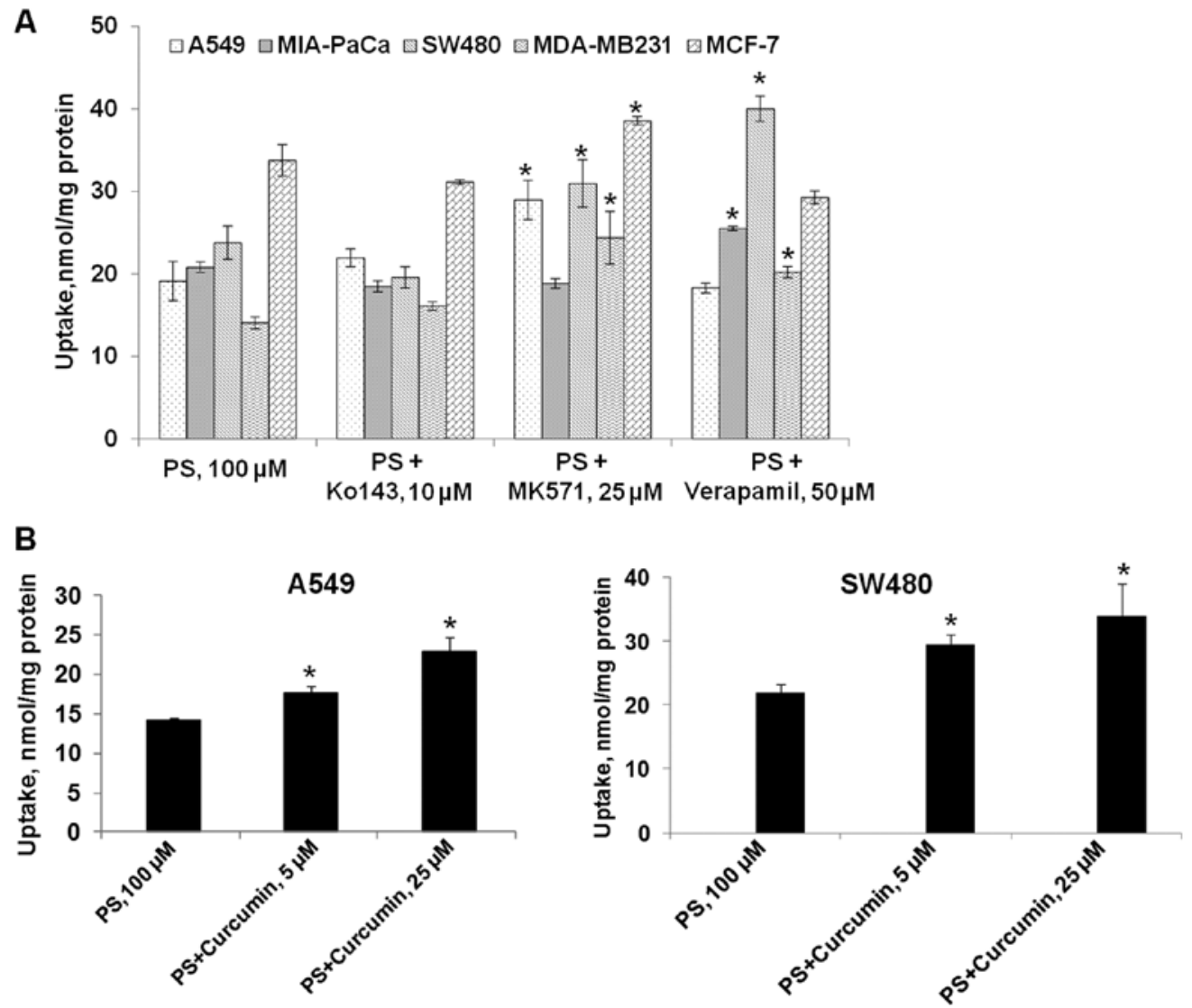

Figure 2. Curcumin and efflux transport inhibitors enhance the cellular uptake of PS. (A) Effect of transporter inhibitors on the cellular uptake of PS. Inhibitor of P-glycoprotein (Verapamil), multidrug resistance protein (MK571) or breast cancer resistance protein (Ko143) was incubated with $100 \mu$ M PS in five human cancer cell lines for $1 \mathrm{~h}$ and the intracellular PS levels were determined by HPLC. (B) Effect of curcumin on cellular uptake of PS. Curcumin (5 or $25 \mu \mathrm{M})$ was co-added with PS in A549 and SW480 cells for $1 \mathrm{~h}$ and the intracellular PS levels were determined by HPLC. Values are mean \pm SEM of at least three independent experiments.
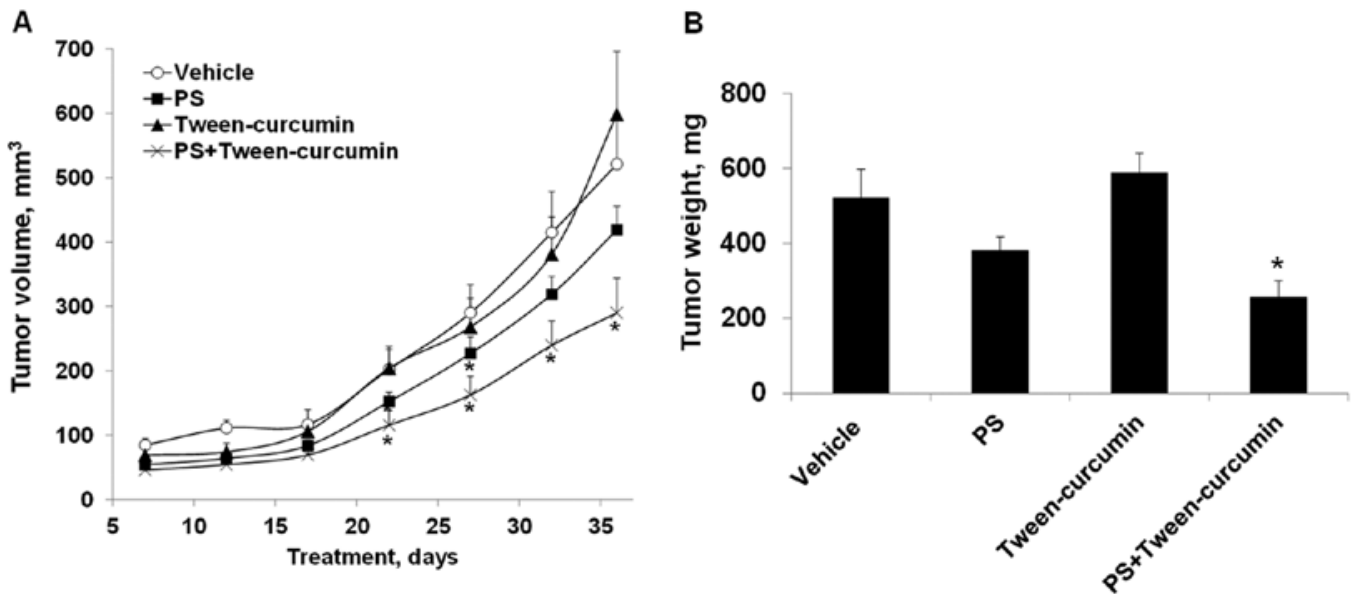

Figure 3. Combination of PS and curcumin synergistically inhibits the growth of A549 lung cancer xenografts in nude mice. (A) Nude mice (n=6) were pre-treated with vehicle, PS (200 mg/kg), curcumin $(500 \mathrm{mg} / \mathrm{kg}$ in $10 \%$ Tween-80), or a combination of PS (200 mg/kg) and curcumin (500 mg/kg in $10 \%$ Tween-80) for 3 days, after which A549 cells were inoculated subcutaneously into both flanks. Treatment was continued until the end of the study and tumor volume was measured at designated time-points. (B) Tumor weights of the different groups were determined at end-point. Values are mean \pm SEM. * $\mathrm{p}<0.05$ compared to control.

small inhibition of tumor growth that was statistically significant on days 17-27 after tumor implantation; whereas neither formulation of curcumin was effective for the duration of the study. On the other hand, PS in combination with curcumin suspended in $10 \%$ Tween- 80 synergistically inhibited the growth of A549 xenografts and the effect was statistically 
A
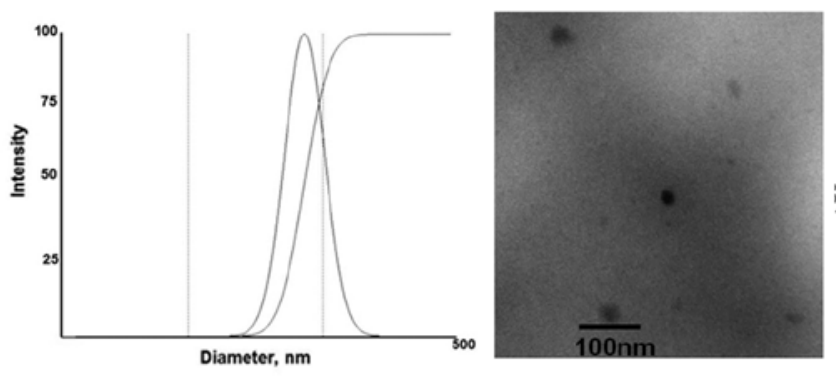

B
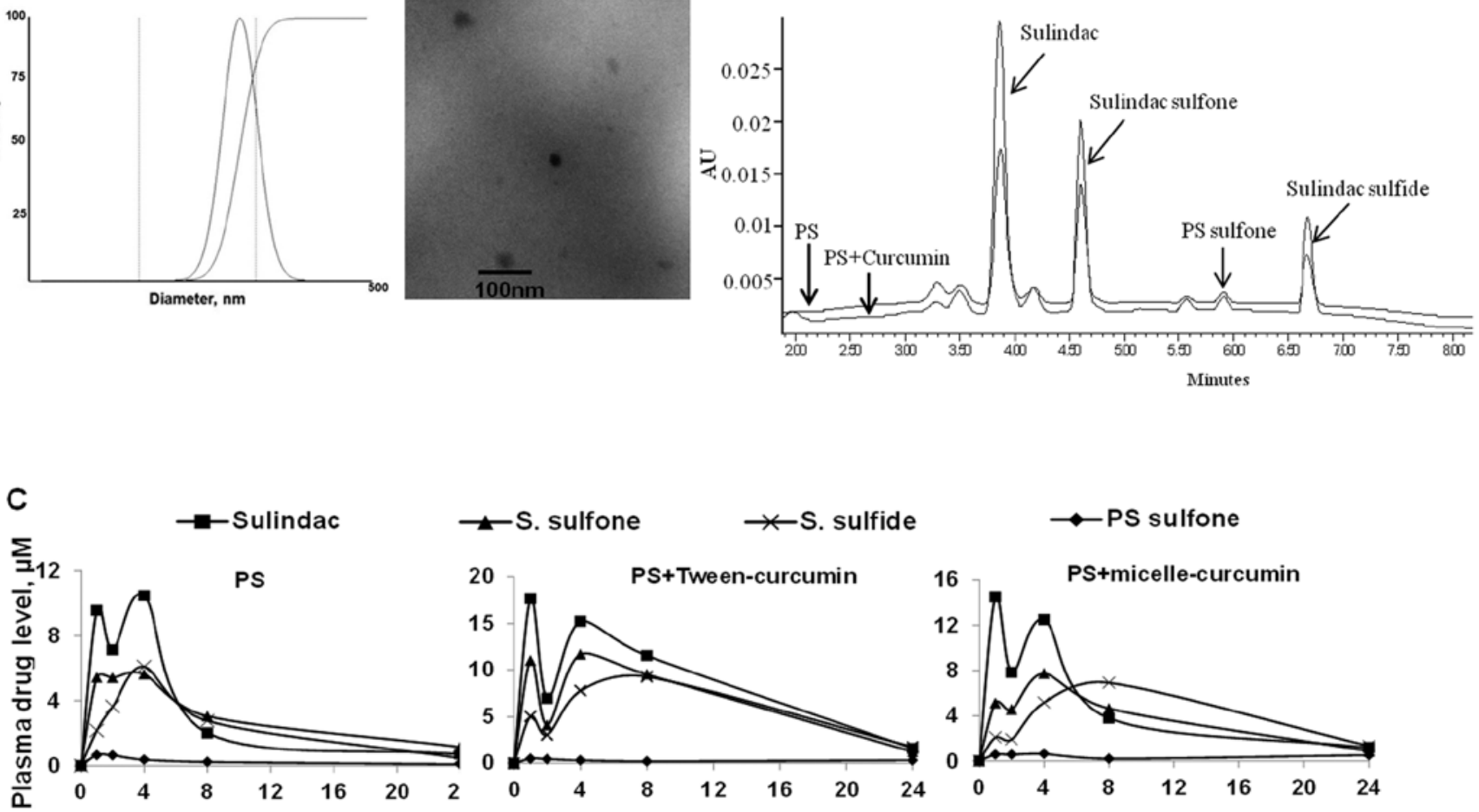

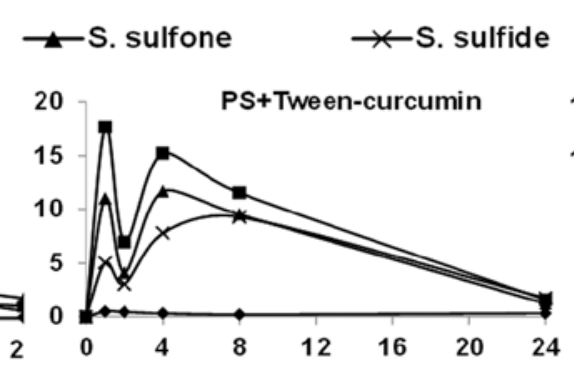

Time post treatment, $\mathrm{h}$

Figure 4. Curcumin enhances the systemic bioavailability of PS in mice. (A) The formulation of curcumin in micelle nanoparticles. The average hydrodynamic diameter (left) and the transmission electron microscopy (TEM) images (right) of curcumin-incorporated micelles. (B) Representative HPLC chromatograms $(328 \mathrm{~nm}$ ) for plasma samples from the PS- and PS-curcumin-treated mice, respectively. PS metabolites are denoted with arrows. (C) In vivo pharmacokinetic study in mice: PS (200 mg/kg), PS (200 mg/kg) plus curcumin (500 mg/kg in 10\% Tween-80), or PS (200 mg/kg) plus curcumin (500 mg/kg in micelles) were administered to mice as a single oral dose and blood samples were collected at the indicated time-points. Plasma levels of the three main PS metabolites, sulindac, sulindac sulfone and sulindac sulfide were determined by HPLC. Values are the average of duplicate samples (all within $15 \%$ of each other).

significant $(\mathrm{p}<0.05)$ beginning on day 12 until the end of the study (day 36). At the end of the study, tumor volume of each group was as follows: control, $521 \pm 76 \mathrm{~mm}^{3}$; PS, $419 \pm 36 \mathrm{~mm}^{3}$; curcumin in $10 \%$ Tween-80: $599 \pm 98 \mathrm{~mm}^{3}$; PS plus curcumin, $290 \pm 54 \mathrm{~mm}^{3}$. This corresponds to a reduction in tumor volume of 19.6 and $44.3 \%$ for PS and PS plus curcumin, respectively. In terms of tumor weight (Fig. 3B), a reduction was observed in the PS $(27 \%, \mathrm{p}=0.06)$ and the PS plus curcumin $(51 \%, \mathrm{p}<0.01)$ groups, but not in the curcumin-treated group. Of note, treatment with PS plus curcumin in $10 \%$ Tween-80 was significantly more effective than PS or curcumin alone $(\mathrm{p}<0.05)$. Surprisingly, no synergistic antitumor activity was observed for the combination between PS and nanoparticles-encapsulated curcumin (data no shown). Taken also into account that PS plus curcumin in 10\% Tween- 80 generated a better PK profile for PS than did PS plus curcumin in nanoparticles, the results of the xenograft study likely suggest the existence of a threshold level for the pharmacological effective dose of PS and/or its metabolites.

PS, curcumin or their combination produced no apparent adverse effects on the mice during the whole duration of the study; and the mean body weights of the treatment groups were comparable to that of the control. At sacrifice, the body weight of the 4 groups of mice was as follows: control, $23 \pm 2 \mathrm{~g}$; PS, $23 \pm 1 \mathrm{~g}$; curcumin, $23 \pm 1 \mathrm{~g}$; and PS plus curcumin, $24 \pm 3 \mathrm{~g}$.
Curcumin improves the bioavailability of PS in mice. Having shown that curcumin enhances the cellular uptake of PS and synergises with PS in inhibiting the growth of human lung cancer xenografts, we next examined the effect of curcumin co-administration on the bioavailability of PS in mice. Curcumin in two formulations, $10 \%$ Tween- 80 or encapsulated in nanoparticles, was given to the mice $30 \mathrm{~min}$ prior to PS administration. As shown in Fig. 4 and Table I, curcumin in both formulations increased the bioavailability of PS in vivo. PS is rapidly metabolized in vivo into several metabolites, of which quantitatively most important are sulindac, sulindac sulfide and sulindac sulfone (23).

Peak plasma levels $\left(\mathrm{C}_{\max }\right)$ of sulindac, sulindac sulfone and sulindac sulfide were much higher after the co-administration of PS with curcumin. The $\mathrm{C}_{\max }$ of sulindac, the main PS metabolite, was increased by $70 \%(17.7 \mu \mathrm{M})$ and $40 \%$ (14.5 vs. $10.4 \mu \mathrm{M}$ for PS alone) when co-administered with curcumin in $10 \%$ Tween-80 or nanoparticles, respectively. As with the case for sulindac, the $\mathrm{C}_{\max }$ of sulindac sulfone and sulindac sulfide were also higher following administration of PS with curcumin. Intact PS, however, was not detected in any of the three treatments, presumably due to the high carboxylesterase activity in the mouse blood (23). In terms of total plasma $\mathrm{AUC}_{0-24 \mathrm{~h}}$, PS given with curcumin in $10 \%$ Tween-80 (500 $\mu \mathrm{M} * \mathrm{~h})$ or nanoparticles $(309 \mu \mathrm{M} * \mathrm{~h})$ increased the sum of $\mathrm{AUC}_{0-24 \mathrm{~h}}$ of all 
Table I. Pharmacokinetic parameters of major metabolites of PS following administration of a single oral dose of PS (200 mg/kg) alone or in combination with curcumin $(500 \mathrm{mg} / \mathrm{kg})$ in $10 \%$ Tween-80 (Tw-curcumin) and micelles (Mic-curcumin), respectively.

\begin{tabular}{|c|c|c|c|c|c|c|c|c|c|}
\hline \multirow[b]{2}{*}{ PS metabolite } & \multicolumn{3}{|c|}{$\mathrm{AUC}_{0-24 \mathrm{~h}}$} & \multicolumn{3}{|c|}{$\mathrm{C}_{\max }, \mu \mathrm{M}$} & \multicolumn{3}{|c|}{$\mathrm{T}_{\max }, \mathrm{h}$} \\
\hline & PS & $\begin{array}{l}\text { PS+Tw- } \\
\text { curcumin }\end{array}$ & $\begin{array}{l}\text { PS+Mic- } \\
\text { curcumin }\end{array}$ & PS & $\begin{array}{l}\text { PS+Tw- } \\
\text { curcumin }\end{array}$ & $\begin{array}{l}\text { PS+Mic- } \\
\text { curcumin }\end{array}$ & PS & $\begin{array}{l}\text { PS+Tw- } \\
\text { curcumin }\end{array}$ & $\begin{array}{l}\text { PS+Mic- } \\
\text { curcumin }\end{array}$ \\
\hline Sulindac & 77.5 & 201.9 & 110.8 & 10.4 & 17.7 & 14.5 & 4 & 1 & 1 \\
\hline Sulindac sulfide & 57.3 & 137.6 & 100.3 & 6.1 & 9.3 & 6.9 & 4 & 8 & 8 \\
\hline Sulindac sulfone & 70.3 & 153.6 & 88.5 & 5.6 & 11.7 & 7.7 & 4 & 4 & 4 \\
\hline PS sulfone & 5.8 & 6.8 & 9.5 & 0.66 & 0.49 & 0.63 & 1 & 1 & 4 \\
\hline
\end{tabular}

A

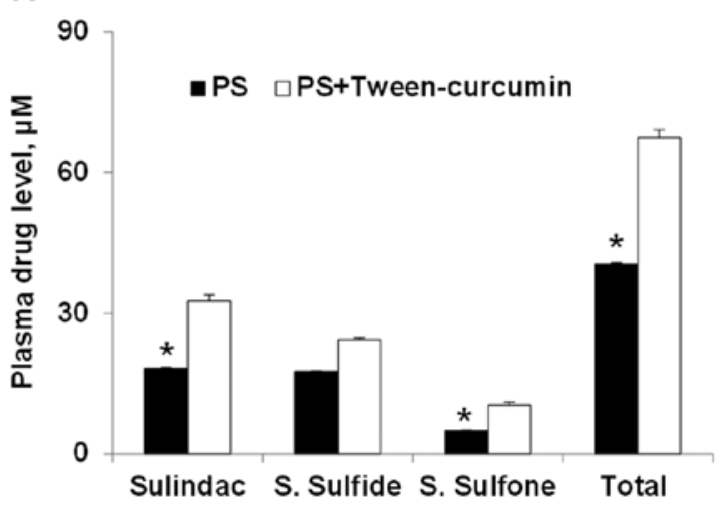

B

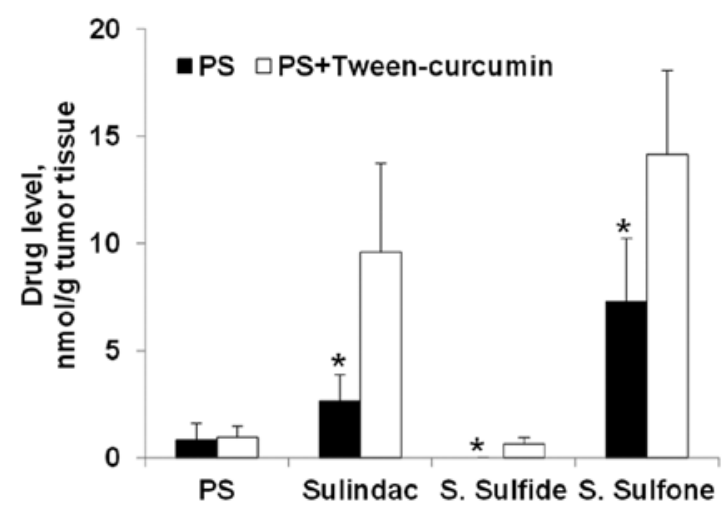

Figure 5. Curcumin co-administration enhances PS levels in tumors. The plasma and tumor levels of PS and its metabolites were measured at the end of the lung cancer xenograft study. (A) Plasma levels of sulindac, sulindac sulfone and sulindac sulfide. (B) Tumor levels of PS and its metabolites. Values are mean \pm SEM. ${ }^{*}$ p $<0.05$ compared to control.

metabolites by 2.4- and 1.5-fold, respectively, compared to PS alone $\left(211 \mu \mathrm{M}^{*} \mathrm{~h}\right)$. Interestingly, curcumin suspended in $10 \%$ Tween- 80 proved to be more effective than the nanoparticle formation in enhancing the pharmacokinetics of PS, giving rise to much higher levels (30-50\%) of PS metabolites. On the other hand, curcumin had no apparent effect on the metabolism of PS in mice, as indicated by their similar metabolic profile (sulindac $>$ sulindac sulfone $=$ sulindac sulfide $>$ PS sulfone). Our results suggest that the co-administration with curcumin enhances the bioavailability of PS without affecting its metabolism in vivo (Fig. 4B and C).

Curcumin enhances PS levels in A549 xenografts. Given the enhanced efficacy of the combined PS and curcumin treatment, we assessed drug levels in the plasma and A549 xenografts from PS, curcumin and the combination treatment groups (Fig. 5). Compared to PS alone, PS plus curcumin generated higher levels of its three main metabolites in the blood. In the A549 xenografts, the levels of sulindac, sulindac sulfide and sulindac sulfone in the PS plus curcumin group were 1-, 3- and 5 -fold higher than those of the PS alone group. The higher levels of PS metabolites are consistent with the higher efficacy achieved with the PS and curcumin combination treatment. We also detected minute levels of curcumin glucuronide in the plasma of curcumin and PS plus curcumin groups, but there was no significant difference in its levels between the two groups. Neither curcumin, nor its glucuronide, was detected in A549 xenografts.

\section{Discussion}

Our study demonstrates that curcumin enhances the efficacy of PS against human lung cancer in pre-clinical models. We establish that curcumin: a) potentiates the cytotoxicity of PS in vitro; b) increases the cellular uptake of PS in cancer cells; c) improves the systemic bioavailability of PS and its metabolites; and d) enhances the delivery of PS and its metabolites to the A549 xenografts, leading to their synergistic growth inhibition by the two agents.

The combination of PS and curcumin suspended in $10 \%$ Tween-80 exerted a strong inhibitory effect on A549 xenografts in nude mice, reducing tumor volume by $44 \%$ and tumor weight by $51 \%$ (both $\mathrm{p}<0.05)$. In contrast, PS $(20 \%$ inhibition) or curcumin (no inhibition) alone did not produce a significant inhibitory effect on A549 xenografts. A key finding of this study is that curcumin co-administration improves the bioavailability and pharmacokinetic properties of PS, as illustrated by higher peak levels $\left(\mathrm{C}_{\text {max }}\right)$ and a 2.4-fold increase in total $\mathrm{AUC}_{0-24 \mathrm{~h}}$ of PS and its metabolites. Consequently, administration of PS plus curcumin resulted in much greater accumulation of PS and its metabolites in A549 xenografts compared to PS alone. The pharmacokinetic profile of 
curcumin, however, was not affected when combined with PS Given that curcumin (and its metabolites) showed no detectable accumulation in tumors and had no tumor inhibitory effect, these data indicate that the synergistic effect of PS plus curcumin in $10 \%$ Tween- 80 is predominantly a consequence of the enhanced delivery of PS and its metabolites to the tumors.

The chemo-sensitizing effect of curcumin is dependent upon its ability to inhibit ATP-binding cassette (ABC) proteins. $\mathrm{ABC}$ transporters are the primary active transporters that mediate the efflux of xenobiotics such as anticancer drugs; and their overexpression in cancer cells is associated with multidrug resistance (24). Curcumin is a promiscuous inhibitor of drug transporters from the ABC family, including P-glycoprotein (MDR1/ABCB1) (25), BCRP (ABCG2) (26) and multiple MRPs [MRP1/ABCC1, MRP2/ABCC2 (27,28), MRP5/ABCC5 (29)]. Our study revealed that curcumin enhances the bioavailability of PS in vitro and in vivo by improving the cellular uptake of PS. The uptake of PS into lung and colon cancer cells in vitro is influenced by drug efflux transporters, such as MRPs and P-glycoproteins, which decrease the accumulation and cellular toxicity of PS. Curcumin, at non-cytotoxic levels, antagonizes the effect of these transporters and thus increases the cellular uptake of PS in cancer cells, thereby potentiating its cytotoxic activity in vitro.

Curcumin may improve the bioavailability of PS in two ways. First, curcumin may inhibit efflux transporters in the intestinal barrier, thus enhancing the absorption of PS. Second, curcumin may also inhibit drug efflux in tumor xenografts, resulting in increased biodistribution of PS and its metabolites to the target tissue. The higher levels of PS and its metabolites in the A549 xenografts were consequential, as they correlated with reduced tumor volume. On the other hand, curcumin did not affect the metabolism of PS by carboxylesterases and cytochrome P450s. Our findings support the idea that curcumin potentiates the antitumor activity of PS through enhanced delivery of PS and its metabolites to tumors. The three quantitatively important metabolites of PS (sulindac, sulindac sulfide and sulindac sulfone) are known to have anticancer properties (23) both through COX-dependent and -independent pathway (30).

In conclusion, our data demonstrate that the co-administration of PS and curcumin synergistically inhibits the growth of human lung cancer xenografts in nude mice. The enhanced efficacy is attributed to inhibition of efflux transporters by curcumin, leading to improved PS bioavailability including the target tumor. This promising drug combination merits further evaluation.

\section{Acknowledgements}

This study was supported by National Institutes of Health Grants HHSN261201000109C, R01 CA101019 and R01 CA139454 and DOD Grants W81XWH 11-1-0799, W81XWH-0710171 and W81XWH1010873.

\section{References}

1. Elwood PC, Gallagher AM, Duthie GG, Mur LA and Morgan G: Aspirin, salicylates and cancer. Lancet 373: 13011309, 2009.
2. Rothwell PM, Price JF, Fowkes FG, et al: Short-term effects of daily aspirin on cancer incidence, mortality and non-vascular death: analysis of the time course of risks and benefits in 51 randomised controlled trials. Lancet 379: 1602-1612, 2012.

3. Rothwell PM, Wilson M, Price JF, et al: Effect of daily aspirin on risk of cancer metastasis: a study of incident cancers during randomised controlled trials. Lancet 379: 1591-1601, 2012.

4. Cheng KW, Mattheolabakis G, Wong CC, et al: Topical phosphosulindac (OXT-328) is effective in the treatment of non-melanoma skin cancer. Int J Oncol 41: 1199-1203, 2012.

5. Huang L, Mackenzie GG, Sun Y, et al: Chemotherapeutic properties of phospho-nonsteroidal anti-inflammatory drugs, a new class of anticancer compounds. Cancer Res 71: 76177627,2011

6. Mackenzie GG, Ouyang N, Xie G, et al: Phospho-sulindac (OXT-328) combined with difluoromethylornithine prevents colon cancer in mice. Cancer Prev Res 4: 1052-1060, 2011.

7. Zhu C, Cheng KW, Ouyang N, et al: Phosphosulindac (OXT-328) selectively targets breast cancer stem cells in vitro and in human breast cancer xenografts. Stem Cells 30: 2065-2075, 2012.

8. Zhu R, Cheng KW, Mackenzie G, et al: Phospho-sulindac (OXT-328) inhibits the growth of human lung cancer xenografts in mice: enhanced efficacy and mitochondria targeting by its formulation in solid lipid nanoparticles. Pharm Res 29: 3090-3101, 2012

9. Hanif R, Qiao L, Shiff SJ and Rigas B: Curcumin, a natural plant phenolic food additive, inhibits cell proliferation and induces cell cycle changes in colon adenocarcinoma cell lines by a prostaglandin-independent pathway. J Lab Clin Med 130: 576-584, 1997.

10. Kawamori T, Lubet R, Steele VE, et al: Chemopreventive effect of curcumin, a naturally occurring anti-inflammatory agent, during the promotion/progression stages of colon cancer. Cancer Res 59: 597-601, 1999.

11. Villegas I, Sanchez-Fidalgo S and de la Lastra CA: Chemopreventive effect of dietary curcumin on inflammation-induced colorectal carcinogenesis in mice. Mol Nutr Food Res 55: 259-267, 2011.

12. Su CC, Yang JS, Lu CC, et al: Curcumin inhibits human lung large cell carcinoma cancer tumour growth in a murine xenograft model. Phytother Res 24: 189-192, 2010.

13. Dance-Barnes ST, Kock ND, Moore JE, et al: Lung tumor promotion by curcumin. Carcinogenesis 30: 1016-1023, 2009.

14. Giladi N, Kazanov D, Shpitz B, Aroch I, Kraus S and Arber N: Curcumin potentiates the pro-apoptotic effects of sulindac sulfone in colorectal cancer. Expert Opin Investig Drugs 19: S117-S124, 2010.

15. Lee JY, Lee YM, Chang GC, et al: Curcumin induces EGFR degradation in lung adenocarcinoma and modulates p38 activation in intestine: the versatile adjuvant for gefitinib therapy. PloS One 6: e23756, 2011.

16. Yin H, Guo R, Xu Y, et al: Synergistic antitumor efficiency of docetaxel and curcumin against lung cancer. Acta Biochim Biophys Sin 44: 147-153, 2012.

17. Ganta S and Amiji M: Coadministration of Paclitaxel and curcumin in nanoemulsion formulations to overcome multidrug resistance in tumor cells. Mol Pharm 6: 928-939, 2009.

18. Sung B, Kunnumakkara AB, Sethi G, et al: Curcumin circumvents chemoresistance in vitro and potentiates the effect of thalidomide and bortezomib against human multiple myeloma in nude mice model. Mol Cancer Ther 8: 959-970, 2009.

19. Francois G and Katz JL: Nanoparticles and nanocapsules created using the Ouzo effect: spontaneous emulisification as an alternative to ultrasonic and high-shear devices. Chemphyschem 6: 209-216, 2005.

20. Galindo-Rodriguez S, Allemann E, Fessi H and Doelker E: Physicochemical parameters associated with nanoparticle formation in the salting-out, emulsification-diffusion and nanoprecipitation methods. Pharm Res 21: 1428-1439, 2004.

21. Wong CC, Cheng KW, Xie G, et al: Carboxylesterases 1 and 2 hydrolyze phospho-nonsteroidal anti-inflammatory drugs: relevance to their pharmacological activity. J Pharmacol Exp Ther 340: 422-432, 2012.

22. Balimane PV, Han $\mathrm{YH}$ and Chong S: Current industrial practices of assessing permeability and P-glycoprotein interaction. AAPS J 8: E1-E13, 2006.

23. Xie G, Nie T, Mackenzie GG, et al: The metabolism and pharmacokinetics of phospho-sulindac (OXT-328) and the effect of difluoromethylornithine. Br J Pharmacol 165: 21522166, 2012. 
24. Gottesman MM, Fojo T and Bates SE: Multidrug resistance in cancer: role of ATP-dependent transporters. Nat Rev Cancer 2: $48-58,2002$.

25. Chearwae W, Anuchapreeda S, Nandigama K, Ambudkar SV and Limtrakul P: Biochemical mechanism of modulation of human P-glycoprotein (ABCB1) by curcumin I, II and III purified from Turmeric powder. Biochem Pharmacol 68: 2043-2052, 2004.

26. Shukla S, Zaher H, Hartz A, et al: Curcumin inhibits the activity of ABCG2/BCRP1, a multidrug resistance-linked ABC drug transporter in mice. Pharm Res 26: 480-487, 2009.

27. Wortelboer HM, Usta M, van der Velde AE, et al: Interplay between MRP inhibition and metabolism of MRP inhibitors: the case of curcumin. Chem Res Toxicol 16: 1642-1651, 2003.
28. Wortelboer HM, Usta M, van Zanden JJ, et al: Inhibition of multidrug resistance proteins MRP1 and MRP2 by a series of alpha,beta-unsaturated carbonyl compounds. Biochem Pharmacol 69: 1879-1890, 2005.

29. Li Y, Revalde JL, Reid G and Paxton JW: Modulatory effects of curcumin on multi-drug resistance-associated protein 5 in pancreatic cancer cells. Cancer Chemother Pharmacol 68: 603-610, 2011.

30. Rigas B and Shiff SJ: Nonsteroidal anti-inflammatory drugs (NSAIDs), cyclooxygenases and the cell cycle. Their interactions in colon cancer. Adv Exp Med Biol 470: 119-126, 1999. 